\title{
Upcrossing Rates for Order Statistics Processes
}

\author{
M. T. Alodat ${ }^{1}$ and M. Al-Rawwash ${ }^{2}$ \\ ${ }^{1}$ Mathematics, Statistics and Physics, Qatar University, Doha, Qatar \\ ${ }^{2}$ Mathematics, Sharjah University, UAE
}

\begin{abstract}
In this paper, we define new random processes as the pointwise order statistics of a set of random processes. We derive the upcrossing rates of the order statistics processes in closed form. Also, we establish relations between the average duration of the order statistics processes and the average duration of their component processes. Finally, we obtain the asymptotic distribution of the excess heights of the new processes.
\end{abstract}

Zusammenfassung: In dieser Arbeit definieren wir neue Zufallsprozesse als die punktweisen Ordnungsstatistiken einer Reihe von Zufallsprozessen. Wir erhalten die Upcrossing Raten der Ordnungsstatistiken in geschlossener Form. Auch schaffen wir die Beziehungen zwischen der durchschnittlichen Dauer der Ordnungsstatistikprozesse und die durchschnittliche Dauer der Teilprozesse. Schließlich erhalten wir die asymptotische Verteilung der ExcessHöhen der neuen Prozesse.

Keywords: Gaussian Process, Order Statistics Process, Duration Distributions, Upcrossing Rate.

\section{Introduction}

The flexibility and attractive theoretical results for Gaussian processes motivate researchers to explore their various properties and highlight the significance of applications of such processes. Among others, Gaussian processes are used to solve regression and classification problems in machine learning and a typical option in digital communication systems is to use Gaussian processes to characterize the noise component. However, while Gaussian processes are promising candidates for solving many real life problems, they do not provide a universal recipe in all situations and non-Gaussian processes are frequently required in engineering, physics and communication studies. This research was motivated by a problem in electrical engineering, where the probability of having two deep fades from independent signals, during the same time interval, is small. The quality of the wireless communication systems and the search for sustainable service provider in such areas motivate engineers to look for solutions that will handle the fading problem. The fading effect can be diminished by combining signals and one of the well-known simple methods used here is the so called selection combining. This method works when $n$ branches are used in receivers and the current value of signal-to-noise ratio on all $n$ branches is estimated by picking the one with highest value (Sagias, Zogas, and Karagiannidis, 2005). In such practical problems, the derivation of the fade averages requires the derivation of the upcrossing rates of order statistics processes. To this end, we introduce the order statistics process based on independent stationary processes $X_{1}(t), \ldots, X_{n}(t)$ and define new 
processes $Y_{1}(t), \ldots, Y_{n}(t)$, called the order statistics processes, such that $Y_{i}(t)$ represents the pointwise $i$ th order statistics of $X_{1}(t), \ldots, X_{n}(t), i=1, \ldots, n$.

To address the aforementioned issues, this article derives the upcrossings rates of the order statistics processes and elaborates on the average duration of these order statistics processes. The situation where the processes are dependent will not be discussed in this article, due to two reasons. First, assuming the dependence of the processes $X_{1}(t), \ldots, X_{n}(t)$ leads to serious technical difficulties, and second, in most important applications independence can be achieved by design. The rest of the article is organized as follows. In Section 2, we review some preliminary results and basic settings. In Section 3, we present the main results regarding the upcrossing rates of the order statistics processes. We also establish some relationships between the upcrossings rates of the processes and their durations. In Section 4, we derive the asymptotic distribution of the excess height for the order statistics processes when the components $X_{1}(t), \ldots, X_{n}(t)$ are Gaussian processes.

\section{Preliminary Results and Basic Settings}

Consider a random process $\{Z(t), t \in[0, A]\}$, where $A>0$, and define $\mu_{Z}(t)=\mathrm{E}(Z(t))$ as the mean function of the process provided that $\mathrm{E}|Z(t)|<\infty$. Also, let $\mathcal{R}_{Z}(t, s)=$ $\operatorname{cov}(Z(t), Z(s))$ denote the covariance function provided that $\mathrm{E}|Z(t)|^{2}<\infty$. Clearly, if $Z(t)$ is stationary then we have $\mathcal{R}_{Z}(t, s)=\mathcal{R}_{Z}(t-s)$. According to Adler (1981) a stationary random process $Z(t)$ admits almost surely continuously differentiable sample paths if its covariance function $\mathcal{R}_{Z}(t)$ has the form

$$
\mathcal{R}_{Z}(h)=\mathcal{R}_{Z}(0)-\frac{1}{2} \lambda h^{2}+o\left(h^{2}\right), \quad \text { as } h \rightarrow 0,
$$

where $\lambda$ is a constant.

Definition 1. A stationary and continuous random process $Z(t), t \in[0, A], A>0$, is said to have an upcrossing of level $u$ at $t_{0} \in[0, A]$ if there exists some $\epsilon>0$ such that $Z(t) \leq u$ for every $t \in\left(t_{0}-\epsilon, t_{0}\right)$ and $Z(t) \geq u$ for every $t \in\left(t_{0}, t_{0}+\epsilon\right)$.

Suppose that $Z(t)$ is a stationary process with continuously differentiable sample paths, let the first derivative of $Z(t)$ be denoted by $\dot{Z}(t)$ and let the probability density function (pdf) $f_{Z(0)}(x)$ be bounded in $x$ in some neighborhood of $u$. Then, according to Bulinskaya's Theorem, $\operatorname{Pr}(\{t \in[0, A]: Z(t)=u$ and $\dot{Z}(t)=0\})=0$ (Leadbetter, Lindgren, and Rootzén, 1983, p. 160). Hence the process $Z(t)$ will have an upcrossing of level $u$ at $t_{0}$ if $Z\left(t_{0}\right)=u$ and $\dot{Z}\left(t_{0}\right)>0$. Similarly, if $Z\left(t_{0}\right)=u$ and $\dot{Z}\left(t_{0}\right)<0$, then $Z(t)$ has a downcrossing of level $u$ at $t_{0}$. In this paper, we shall assume that all processes used to define the order statistic process satisfy the conditions of Bulinskaya's Theorem.

Random processes and random fields are vital in engineering, physics, and medical fields. In particular, the supremum of a random process, namely $\sup _{t \in[0, A]} Z(t)$ is very relevant in such applications. Therefore, the need to derive its distribution is imminent and it leads to several probabilistic problems about the phenomenon of interest. Alodat and 
Anagreh (2011) discussed the upcrossing rates of random processes and the duration distribution of the Rayleigh process. They discussed the application of wind turbines where the wind is converted to clean energy and eventually produces electricity. Usually, the turbine system should automatically stop generating electricity if the wind speed assumes extreme values. If the wind speed is modeled by a stochastic process $Z(t)$, the probability that the wind speed exceeds a given threshold $u$ is given by $\operatorname{Pr}\left(\sup _{t \in[0, A]} Z(t)>u\right)$. In general it is very difficult to derive this distribution (sometimes called excursion probability), except for a very limited number of cases. On the other hand, crossing methods are alternatives that can be used to study such a distribution. To accomplish this, we use an approximation introduced in Adler and Taylor (2007) and implement this method for $\operatorname{Pr}\left(\sup _{t \in[0, A]} Z(t)>u\right)$ assuming sufficiently large $u$ and $A$ which allows us to conclude that

$$
\operatorname{Pr}\left(\sup _{t \in[0, A]} Z(t)>u\right) \approx \mathrm{E}(N(u, A, Z)),
$$

where $N(u, A, Z)$ denotes the number of upcrossings of level $u$ of $Z(t)$ in $[0, A]$. Having argued the need of the supremum of the random process and the valid approximation of the supremum value distribution of $Z(t)$ given in (2), we may obtain such distribution through the computation of the expected number of upcrossings. We plan to use Rice's Formula (Aldous, 1989) to derive the expected number of upcrossings for an almost surely differentiable and stationary random process $Z(t)$ as

$$
\mathrm{E}(N(u, A, Z))=A \mathrm{E}\left(\dot{Z}(0)^{+} \mid Z(0)=u\right) f_{Z(0)}(u),
$$

where $x^{+}=\max (0, x)$ and $f_{Z(0)}(u)$ is the pdf of $Z(0)$. For a zero-mean stationary Gaussian process, the expected number of upcrossings in (3) reduces to

$$
\mathrm{E}(N(u, A, Z))=A \frac{\lambda^{1 / 2}}{2 \pi \sigma} \exp \left(-\frac{u^{2}}{2 \sigma^{2}}\right)
$$

where $\sigma^{2}=\operatorname{var}(Z(0))$ and $\lambda=\operatorname{var}(\dot{Z}(0))$.

The set of all values of $t \in[0, A]$ for which the process $Z(t)$ exceeds the threshold $u$ is called the excursion set of $Z(t)$ and it is of central interest in several applications of random processes. Probabilists define the excursion set of the process $Z(t), t \in[0, A]$, above the level $u$ as $\xi(u, A, Z)=\{t \in[0, A]: Z(t) \geq u\}$. Excursion sets are discussed in different occasions in the literature (Adler, 1981; Worsley, 1994; Alodat, Al-Rawwash, and Jebrini, 2010). For a wide class of smooth random processes, the excursion set decomposes into a finite union of disjoint intervals (Aldous, 1989) as $u \rightarrow \infty$. The length of each interval in such a decomposition is called a duration of $Z(t)$ above $u$. Furthermore, asymptotically these durations are independent and identically distributed such that $N(u, A, Z)$ follows a Poisson distribution. In term of upcrossings, the duration of $Z(t)$ is defined as the length of the interval between an upcrossing and the subsequent downcrossing of level $u$. Since these durations behave independently and are identically distributed, we use a common notation $S_{Z}$ to denote a duration of $Z(t)$ in $[0, A]$ above $u$. Assume that we have a duration $S_{Z}$ generated by a process $Z(t)$ above $u$, the mean of $S_{Z}$ is obtained via the following Poisson Clumping Heuristic formula (Aldous, 1989)

$$
\mathrm{E}\left(S_{Z}\right)=\frac{A \operatorname{Pr}(Z(0)>u)}{\mathrm{E}(N(u, A, Z))}
$$


If the process $Z(t)$ satisfies $\{0, A\} \notin \xi(u, A, Z)$, then $\mathrm{E}(N(u, A, Z))$ equals the average number of durations. Hence $\mathrm{E}\left(S_{Z}\right)$ is simply the ratio of the average length of the excursion set and the average number of up crossings/durations. Moreover, if the process $Z(t)$ satisfies the condition $\{0, A\} \notin \xi(u, A, Z)$ with small probability (which occurs for large values of $u)$, then $\mathrm{E}(N(u, A, Z))$ approximates the average number of durations.

The mean duration (sometimes called average level crossing rate or average fade) of a random process has been used for a long time in the communication engineering literature to study the quality and the efficiency of wireless communication systems. For example, an average fading of 30 to $40 \mathrm{~dB}$ may take place several times in a given second, depending on the speed of the mobile unit and the carrier frequency (Stefanović, Panić, Stefanović, Nikolić, and Cvetković, 2012). Because of the random nature of such events, researchers concluded that the signal of a digital telecommunication system can be viewed as a random process. Due to multipath wave propagation, wireless communication systems are subjected to strong fading influences. In order to reduce the fading effects, the so called selection combining (SC) is considered as a common option to analyze signals from different paths. The SC picks the strongest one among the received signals. If $X_{1}(t), \ldots, X_{n}(t)$ denote the values of the signals from $n$ branches at time $t$, then the receiver uses the best one defined as $Y_{n}(t)=\max \left\{X_{1}(t), \ldots, X_{n}(t)\right\}$. In this case the average fading of the SC signal is

$$
\text { Average fade of } Y_{n}(t)=\frac{A \operatorname{Pr}\left(Y_{n}(0)>u\right)}{\mathrm{E}\left(N\left(u, A, Y_{n}\right)\right)} .
$$

The derivation of the upcrossing rate of a process plays a central role in many applications including wireless communication systems via the average fade. As a result, we focus here on generalizing these results to any order statistics process.

It is worth to note that $Y_{1}(t), \ldots, Y_{n}(t)$ are not necessarily differentiable functions in $t$. Therefore, the upcrossing rates of $Y_{1}(t), \ldots, Y_{n}(t)$ can be derived according to Leadbetter and Spaniolo (2002). For an almost sure continuous process $Z(t), t \in[0, A]$, we have

$$
\mathrm{E}(N(u, A, Z))=A \lim _{h \downarrow 0} J_{h}(u, A, Z)
$$

where $J_{h}(u, A, Z)=h^{-1} \operatorname{Pr}(Z(0)<u<Z(h))$. Let $Z(t)$ and $W(t), t \in[0, A]$, be two stationary and ergodic processes such that $Z(t)$ has a continuous marginal distribution, then the distribution of $W(t)$ after an upcrossing of $u$ by $Z(t)$ is given by

$$
G(y)=\frac{\mathrm{E}(N(u, y, A, Z, W))}{\mathrm{E}(N(u, A, Z))}, \quad y>0
$$

where $N(u, y, A, Z, W)$ is the number of upcrossings of level $u$ by the process $Z(t)$ such that $W\left(t_{k}\right) \leq y, t_{k} \in(0, A)$. We clearly see that $G(y)$ does not depend on $A$ and the expected value of $N(u, y, A, Z, W)$ is obtained as

$$
\mathrm{E}(N(u, y, A, Z, W))=A \lim _{h \downarrow 0} J_{h}(u, y, A, Z, W),
$$

where $J_{h}(u, y, A, Z, W)=h^{-1} \operatorname{Pr}(Z(0)<u<Z(h), W(0) \leq y)$. 


\section{Main Results}

In this section we present the main findings concerning the expected number of upcrossings of level $u$ by the order statistics of a collection of independent and stationary processes $X_{1}(t), \ldots, X_{n}(t), t \in[0, A]$. To this end, we consider $Y_{i}(t)$ being the $i$ th order statistics of the processes $X_{1}(t), \ldots, X_{n}(t)$ and we present the following results.

Theorem 1. Let $X_{i}(t), i=1, \ldots, n, t \in[0, A]$, denote independent and stationary processes with cumulative distribution functions $F_{X_{i}(0)}(x)$, then

$$
\mathrm{E}\left(N\left(u, y, A, Y_{1}, Y_{n}\right)\right)=\prod_{i=1}^{n}\left(F_{X_{i}(0)}(y)-F_{X_{i}(0)}(u)\right) \times \sum_{j=1}^{n} \frac{\mathrm{E}\left(N\left(u, A, X_{j}\right)\right)}{F_{X_{j}(0)}(y)-F_{X_{j}(0)}(u)} .
$$

Proof. First we consider $\operatorname{Pr}\left(Y_{1}(0)<u<Y_{1}(h), Y_{n}(0) \leq y\right)$ and rewrite it as

$$
\begin{aligned}
& =\operatorname{Pr}\left(Y_{1}(h)>u, Y_{n}(0) \leq y\right)-\operatorname{Pr}\left(Y_{1}(0)>u, Y_{1}(h)>u, Y_{n}(0) \leq y\right) \\
& =\prod_{i=1}^{n} \operatorname{Pr}\left(u<X_{i}(h), X_{i}(0) \leq y\right)-\prod_{i=1}^{n} \operatorname{Pr}\left(X_{i}(0)>u, u<X_{i}(h), X_{i}(0) \leq y\right) \\
& =\prod_{i=1}^{n} \operatorname{Pr}\left(u<X_{i}(h), X_{i}(0) \leq y\right) \\
& \quad \quad-\prod_{i=1}^{n} \operatorname{Pr}\left(u<X_{i}(h), X_{i}(0) \leq y\right) \times \prod_{j=1}^{n}\left(1-h \frac{J_{h}\left(u, A, X_{j}\right)}{\operatorname{Pr}\left(u<X_{j}(h), X_{j}(0) \leq y\right)}\right) \\
& =h \prod_{i=1}^{n} \operatorname{Pr}\left(u<X_{i}(h), X_{i}(0) \leq y\right) \times \sum_{j=1}^{n} \frac{J_{h}\left(u, A, X_{j}\right)}{\operatorname{Pr}\left(u<X_{j}(h), X_{j}(0) \leq y\right)}+o\left(h^{2}\right) .
\end{aligned}
$$

Finally, dividing this identity by $h$ and taking the limit as $h \downarrow 0$ concludes the proof.

Assuming that $X_{i}(t)$ is an ergodic process and implementing the result of Theorem 1, then for $y>0$ we may write (7) as

$$
G(y)=\frac{\prod_{i=1}^{n}\left(F_{X_{i}(0)}(y)-F_{X_{i}(0)}(u)\right) \times \sum_{j=1}^{n} \frac{\mathrm{E}\left(N\left(u, A, X_{j}\right)\right)}{F_{X_{j}(0)}(y)-F_{X_{j}(0)}(u)}}{\prod_{i=1}^{n}\left(1-F_{X_{i}(0)}(u)\right) \times \sum_{j=1}^{n} \frac{\mathrm{E}\left(N\left(u, A, X_{j}\right)\right)}{1-F_{X_{j}(0)}(u)}} .
$$

The function $G(y)$ represents the cumulative distribution function (cdf) of the process $Y_{n}(t)$ after an upcrossing of level $u$ by the process $Y_{1}(t)$. In wireless communication industry, engineers frequently refer to the quantity $(1-G(y))$ which represents the probability that the largest signal value exceeds a threshold $y$ after an upcrossing of level $u$ by the smallest (weakest) signal. In the following theorem we establish two relationships between $\mathrm{E}\left(N\left(u, A, Y_{1}\right)\right), \ldots, \mathrm{E}\left(N\left(u, A, Y_{n}\right)\right)$ and $\mathrm{E}\left(N\left(u, A, X_{1}\right)\right)$.

Theorem 2. Let the processes $X_{1}(t), \ldots, X_{n}(t)$ be independent and identically distributed with common cdf $F(u)$, then 
(a) The mean of the number of upcrossings of $u$ by $Y_{i}(t)$ is

$$
\mathrm{E}\left(N\left(u, A, Y_{i}\right)\right)=\frac{n !}{(i-1) !(n-i) !} F(u)^{i-1}(1-F(u))^{n-i} \mathrm{E}\left(N\left(u, A, X_{1}\right)\right) .
$$

(b) $\mathrm{E}\left(N\left(u, A, X_{1}\right)\right)=\frac{1}{n} \sum_{i=1}^{n} \mathrm{E}\left(N\left(u, A, Y_{i}\right)\right)$.

Proof. We outline the proof of part (a) while part (b) is left for the reader since it is a straightforward result. For each $t$ the $i$ th order statistic $Y_{i}(t)$ of $X_{1}(t), \ldots, X_{n}(t)$ is stationary but not differentiable and we plan to use equation $(6)$ to find its upcrossings mean. To achieve that, we first notice that

$$
\operatorname{Pr}\left(Y_{i}(0)<u<Y_{i}(h)\right)=\operatorname{Pr}\left(Y_{i}(0)<u\right)-\operatorname{Pr}\left(Y_{i}(0)<u, Y_{i}(h)<u\right),
$$

where

$$
\operatorname{Pr}\left(Y_{i}(0)<u\right)=\sum_{j=i}^{n}\left(\begin{array}{c}
n \\
j
\end{array}\right) F(u)^{j}(1-F(u))^{n-j} .
$$

On the other hand, the second term of (9) is

$$
\operatorname{Pr}\left(Y_{i}(0)<u, Y_{i}(h)<u\right)=\operatorname{Pr}\left(\begin{array}{c}
\text { at least } i \text { of } X_{1}(0), \ldots, X_{n}(0) \text { are less than } u \\
\text { and } \\
\text { at least } i \text { of } X_{1}(h), \ldots, X_{n}(h) \text { are less than } u
\end{array}\right) .
$$

Define the variables $Z_{1}$ : the number of $X_{i}(0)$ 's that are less than $u, Z_{2}$ : the number of $X_{i}(h)$ 's that are less than $u, Z_{3}$ : the number of $X_{i}(0)$ 's that are greater than or equal to $u$, and $Z_{4}$ : the number of $X_{i}(h)$ 's that are greater than or equal to $u$. Hence,

$$
\begin{aligned}
\operatorname{Pr}\left(Y_{i}(0)<u, u<Y_{i}(h)\right)= & \sum_{r=i}^{n} \sum_{s=i}^{n} \operatorname{Pr}\left(Z_{1}=r, Z_{2}=s, Z_{3}=n-r, Z_{4}=n-s\right) \\
= & \sum_{r=i}^{n} \operatorname{Pr}\left(Z_{1}=r, Z_{2}=n-r\right) \\
& +\sum_{\substack{r=i \\
s \neq r}}^{n} \sum_{\substack{s=i \\
s \neq r}}^{n} \operatorname{Pr}\left(Z_{1}=r, Z_{2}=s, Z_{3}=n-r, Z_{4}=n-s\right) .
\end{aligned}
$$

We notice that the event $\left\{Z_{1}=r, Z_{2}=s, Z_{3}=n-r, Z_{4}=n-s\right\}$ is a subset of the event $\left\{Z_{1}=r, Z_{4}=n-s\right\}$. Hence,

$$
\begin{aligned}
\operatorname{Pr}\left(Z_{1}=r, Z_{2}\right. & \left.=s, Z_{3}=n-r, Z_{4}=n-s\right) \leq \operatorname{Pr}\left(Z_{1}=r, Z_{4}=n-s\right) \\
= & \left(\begin{array}{l}
n \\
k
\end{array}\right) \operatorname{Pr}\left(X_{i}(0)<u<X_{i}(h)\right)^{k}\left(1-\operatorname{Pr}\left(X_{i}(0)<u<X_{i}(h)\right)\right)^{n-k}=g(h),
\end{aligned}
$$

where $k=\min (r, s)$ and the function $g(h)$ is $o(h)$. On the other hand,

$$
\begin{aligned}
\sum_{r=i}^{n} \operatorname{Pr}\left(Z_{1}\right. & \left.=r, Z_{2}=n-r\right) \\
& =\sum_{r=i}^{n}\left(\begin{array}{l}
n \\
r
\end{array}\right) \operatorname{Pr}\left(X_{i}(0)<u, X_{i}(h)<u\right)^{r}\left(1-\operatorname{Pr}\left(X_{i}(0)>u, X_{i}(h)>u\right)\right)^{n-r} .
\end{aligned}
$$


Applying (6) for the process $X_{i}(t)$ we get

$$
\lim _{h \downarrow 0} h^{-1} \operatorname{Pr}\left(X_{i}(0)<u<X_{i}(h)\right)=\mathrm{E}\left(N\left(u, A, X_{1}\right)\right) .
$$

Since $\operatorname{Pr}\left(X_{i}(0)<u, X_{i}(h)<u\right)=\operatorname{Pr}\left(X_{i}(0)<u\right)-\operatorname{Pr}\left(X_{i}(0)<u<X_{i}(h)\right)$, dividing $-\theta(h)$ by $h$, and taking the limit as $h \downarrow 0$, yields the first derivative of $-\theta(h)$ at $h=0$, i.e.

$$
-\dot{\theta}(0)=\sum_{j=i}^{n}\left(\begin{array}{l}
n \\
j
\end{array}\right)\left(j F(u)^{j-1}+(n-j)(1-F(u))^{n-1-j}\right) \mathrm{E}\left(N\left(u, A, X_{1}\right)\right) .
$$

This simplifies to (7) and completes the proof.

Remark 1: We may estimate the smoothness parameter $\lambda^{1 / 2}$ using Theorem 2(a). If the processes $X_{i}(t)$ are independent and identically distributed Gaussian processes with zero mean and known common variance $\sigma^{2}$, then

$$
\hat{\lambda}^{1 / 2}=\frac{c N\left(u, A, Y_{i}\right)}{i\left(\begin{array}{l}
n \\
i
\end{array}\right) F(u)^{i-1}(1-F(u))^{n-i}}
$$

is an unbiased estimator of $\lambda^{1 / 2}$, where $c=\frac{1}{A} 2 \pi \sigma \exp \left(u^{2} / 2 \sigma^{2}\right)$. According to Leadbetter et al. (1983) and Aldous (1989), the random variable $N\left(u, A, Y_{i}\right)$ has approximately a Poisson distribution as $u \rightarrow \infty$ with mean given by Theorem 2(a). Hence,

$$
\operatorname{var}\left(\hat{\lambda}^{1 / 2}\right) \approx \frac{c}{\left(\begin{array}{c}
n \\
i
\end{array}\right) F(u)^{i-1}(1-F(u))^{n-i}} \lambda^{1 / 2} .
$$

In the sequel, we focus on the marginal cdf of the process $Y_{i}(t)$ when the $X_{i}(t)$ 's are independent but not identically distributed. According to David and Nagaraja (2003) this cdf is

$$
F_{Y_{r}}(x)=\sum_{i=r}^{n} \sum_{S_{i}} \prod_{k=1}^{i} F_{X_{j_{k}}}(x) \prod_{k=i+1}^{n}\left(1-F_{X_{j_{k}}}(x)\right),
$$

where the summation over $S_{i}$ extends over all permutations $\left(j_{1}, \ldots, j_{n}\right)$ of $1, \ldots, n$ such that $j_{1}<\cdots<j_{i}<j_{i+1}<\cdots<j_{n}$. In Theorem 3, we plan to find the expected number of upcrossings by the $r$ th order statistic $Y_{r}(t)$ of $X_{1}(t), \ldots, X_{n}(t)$, which is given by

$$
\mathrm{E}\left(N\left(u, A, Y_{r}\right)\right)=\lim _{h \rightarrow 0} h^{-1} \operatorname{Pr}\left(Y_{r}(0)<u<Y_{r}(h)\right) .
$$

Theorem 3. Let $X_{1}(t), \ldots, X_{n}(t)$ be independent and stationary random processes. Then

$$
\begin{aligned}
\mathrm{E}\left(N\left(u, A, Y_{r}\right)\right)=\sum_{i=r}^{n} \sum_{S_{i}} \prod_{k=1}^{i} F_{X_{j_{k}}}(u) & \prod_{k=i+1}^{n}\left(1-F_{X_{j_{k}}}(u)\right) \\
& \left(\sum_{\nu=1}^{i} \frac{\mathrm{E}\left(N\left(u, A, X_{j_{\nu}}\right)\right)}{F_{X_{j_{\nu}}}(u)}+\sum_{\nu=i+1}^{n} \frac{\mathrm{E}\left(N\left(u, A, X_{j_{\nu}}\right)\right)}{1-F_{X_{j_{\nu}}}(u)}\right) .
\end{aligned}
$$




\section{Proof.}

$$
\begin{aligned}
\operatorname{Pr}\left(Y_{r}(0)<u<Y_{r}(h)\right)=\operatorname{Pr}\left(Y_{r}(0) \leq u\right)-\operatorname{Pr}\left(Y_{r}(0) \leq u, Y_{r}(h) \leq u\right) \\
=F_{Y_{r}}(u)-\sum_{m=r}^{n} \sum_{l=r}^{n} \operatorname{Pr}\left(\text { only } m \text { of } X_{i}(0) \text { 's } \leq u \text {, only } l \text { of } X_{i}(h) \text { 's } \leq u\right) \\
=F_{Y_{r}}(u)-\sum_{l=r}^{n} \operatorname{Pr}\left(\text { only } l \text { of } X_{i}(0) \text { 's } \leq u \text {, only } l \text { of } X_{i}(h) \text { 's } \leq u\right) \\
\quad-\sum_{m=r}^{n} \sum_{l=r}^{n} \operatorname{Pr}\left(\text { at least } m \text { of } X_{i}(0) \text { 's } \leq u \text {, at least } l \text { of } X_{i}(h) \text { 's } \leq u\right) \\
=F_{Y_{r}}(u)-\sum_{i=r}^{n} \sum_{S_{i}} \prod_{k=1}^{i} F_{Z_{j_{k}}}(u) \prod_{k=i+1}^{n}\left(1-F_{Z_{j_{k}}}(u)\right) \\
\quad-\sum_{m=r}^{n} \sum_{l=r}^{n} \operatorname{Pr}\left(\text { only } m \text { of } X_{i}(0) \text { 's } \leq u, \text { only } l \text { of } X_{i}(0) \text { 's } \leq u\right),
\end{aligned}
$$

where $Z_{i}(t)=\max \left(X_{i}(0), X_{i}(h)\right)$, for $i=1, \ldots, n$. Now the second term is

$$
\begin{aligned}
& \sum_{i=r}^{n} \sum_{S_{i}} \prod_{k=1}^{i} F_{Z_{j_{k}}}(u) \prod_{k=i+1}^{n}\left(1-F_{Z_{j_{k}}}(u)\right) \\
& =\sum_{i=r}^{n} \sum_{S_{i}} \prod_{k=1}^{i} \operatorname{Pr}\left(X_{j_{k}}(0)<u, X_{j_{k}}(h)<u\right) \prod_{k=i+1}^{n}\left(1-\operatorname{Pr}\left(X_{j_{k}}(0)<u, X_{j_{k}}(h)<u\right)\right) \\
& =\sum_{i=r}^{n} \sum_{S_{i}} \prod_{k=1}^{i}\left(F_{X_{j_{k}}(0)}(u)-h J_{h}\left(u, A, X_{j_{k}}\right)\right) \prod_{k=i+1}^{n}\left(1-F_{X_{j_{k}}(0)}(u)+h J_{h}\left(u, A, X_{j_{k}}\right)\right) \\
& =\sum_{i=r}^{n} \sum_{S_{i}} \prod_{k=1}^{i} F_{X_{j_{k}}(0)}(u)\left(1-h \frac{J_{h}\left(u, A, X_{j_{k}}\right)}{F_{X_{j_{k}}(0)}(u)}\right) \\
& \quad \times \prod_{k=i+1}^{n}\left(1-F_{X_{j_{k}}(0)}(u)\right)\left(1-h \frac{J_{h}\left(u, A, X_{j_{k}}\right)}{1-F_{X_{j_{k}}(0)}(u)}\right) .
\end{aligned}
$$

Expanding the above product allows us to cancel $F_{Y_{r}}(u)$ from equation (10), which reduces to

$$
\begin{aligned}
-F_{Y_{r}}(u)-h \sum_{i=r}^{n} \sum_{S_{i}} \prod_{k=1}^{i} F_{X_{j_{k}}}(u) & \prod_{k=i+1}^{n}\left(1-F_{X_{j_{k}}}(u)\right) \\
\times & \left(\sum_{\nu=1}^{i} \frac{J_{h}\left(u, A, X_{j_{v}}\right)}{F_{X_{j_{\nu}}}(u)}+\sum_{\nu=i+1}^{n} \frac{J_{h}\left(u, A, X_{j_{v}}\right)}{1-F_{X_{j_{\nu}}}(u)}\right)+o(h) .
\end{aligned}
$$


To finish the proof we notice that the last term can be written as

$$
\begin{aligned}
& \sum_{\substack{m=r \\
l \neq m}}^{n} \sum_{\substack{l=r \\
n}}^{n} \operatorname{Pr}\left(\text { only } m \text { of } X_{i}(0) \text { 's } \leq u \text {, only } l \text { of } X_{i}(h) \text { 's } \leq u\right) \\
& =\sum_{\substack{m=r \\
l \neq m}}^{n} \sum_{\substack{l=r \\
l}}^{n} \prod_{i=1}^{n} \operatorname{Pr}\left(\left(X_{i}(0), X_{i}(h)\right) \in \mathcal{A}_{l m}\right),
\end{aligned}
$$

where the $\mathcal{A}_{l m}$ 's take the form $(-\infty, u] \times(-\infty, u]$ or $[u, \infty) \times(-\infty, u]$. Also

$$
\begin{aligned}
\operatorname{Pr}\left(\left(X_{i}(0), X_{i}(h)\right) \in \mathcal{A}_{l m}\right) & \left.=h \operatorname{Pr}\left(X_{i}(0)<u, X_{i}(h)>u\right) \operatorname{Pr}\left(X_{i}(0)>u, X_{i}(h)<u\right)\right) \\
& \left.=h J_{h}\left(u, A, X_{i}\right) \operatorname{Pr}\left(X_{i}(0)>u, X_{i}(h)<u\right)\right) .
\end{aligned}
$$

Applying the sandwich theorem from an elementary calculus to the right hand side of the last equation, it follows that $\operatorname{Pr}\left(\left(X_{i}(0), X_{i}(h)\right) \in \mathcal{A}_{l m}\right)$ is of order $o(h)$. The result follows by dividing both sides by $h$ and taking the limit as $h \rightarrow 0$.

Corollary 1. The expected numbers of upcrossings of level $u$ by $Y_{1}(t)$ and $Y_{n}(t)$ are given by

(i) $\mathrm{E}\left(N\left(u, A, Y_{1}\right)\right)=\prod_{i=1}^{n}\left(1-F_{X_{i}(0)}(u)\right) \sum_{i=1}^{n} \frac{\mathrm{E}\left(N\left(u, A, X_{i}\right)\right)}{1-F_{X_{i}(0)}(u)}$

(ii) $\mathrm{E}\left(N\left(u, A, Y_{n}\right)\right)=\prod_{i=1}^{n} F_{X_{i}(0)}(u) \sum_{i=1}^{n} \frac{\mathrm{E}\left(N\left(u, A, X_{i}\right)\right)}{F_{X_{i}(0)}(u)}$.

Proof. We prove (ii) and we leave (i) for the reader.

$$
\begin{aligned}
\operatorname{Pr}\left(Y_{n}(0)<u<Y_{n}(h)\right) & =\operatorname{Pr}\left(Y_{n}(0)<u\right)-\operatorname{Pr}\left(Y_{n}(0)<u, Y_{n}(h)<u\right) \\
& =\prod_{i=1}^{n} F_{X_{i}(0)}(u)-\prod_{i=1}^{n}\left(F_{X_{i}(0)}(u)-h J_{h}\left(u, X_{i}, h\right)\right) \\
& =\prod_{i=1}^{n} F_{X_{i}(0)}(u)-\prod_{i=1}^{n} F_{X_{i}(0)}(u) \prod_{i=1}^{n}\left(1-h \frac{J_{h}\left(u, X_{i}, h\right)}{F_{X_{i}(0)}(u)}\right) \\
& =-h \prod_{i=1}^{n} F_{X_{i}(0)}(u) \sum_{j=1}^{n} \frac{J_{h}\left(u, X_{i}, h\right)}{F_{X_{i}(0)}(u)}+o(h) .
\end{aligned}
$$

The result follows by dividing both sides by $h$ and then taking the limit as $h \downarrow 0$.

Corollary 2. If the $X_{i}(t)$ 's are independent and identically distributed then the means of $N\left(u, A, Y_{1}\right)$ and $N\left(u, A, Y_{n}\right)$ are given by

(i) $\mathrm{E}\left(N\left(u, A, Y_{1}\right)\right)=n\left(1-F_{X_{1}(0)}(u)\right)^{n-1} \mathrm{E}\left(N\left(u, A, X_{1}\right)\right)$

(ii) $\mathrm{E}\left(N\left(u, A, Y_{n}\right)\right)=n F_{X_{1}(0)}(u)^{n-1} \mathrm{E}\left(N\left(u, A, X_{1}\right)\right)$.

Theorem 4. The average duration $\mathrm{E}\left(S_{X_{1}}\right)$ of $X_{1}(t)$ can be viewed as a convex linear combination of $\mathrm{E}\left(S_{Y_{1}}\right), \ldots, \mathrm{E}\left(S_{Y_{n}}\right)$, i.e.

$$
\mathrm{E}\left(S_{X_{1}}\right)=\sum_{i=1}^{n} \omega_{i} \mathrm{E}\left(S_{Y_{i}}\right)
$$


where $\omega_{i}=\frac{i}{n}\left(\begin{array}{c}n \\ i\end{array}\right) F(u)^{i-1}(1-F(u))^{n-i}$.

Proof. Let $S_{Y_{i}}$ be the duration of the process $Y_{i}(t)$ above the level $u$ then

$$
\mathrm{E}\left(S_{Y_{i}}\right)=\frac{1-\sum_{j=i}^{n}\left(\begin{array}{c}
n \\
j
\end{array}\right) F(u)^{j}(1-F(u))^{n-j}}{i\left(\begin{array}{c}
n \\
i
\end{array}\right) F(u)^{i-1}(1-F(u))^{n-i} \mathrm{E}\left(N\left(u, A, X_{1}\right)\right)} .
$$

This leads to

$$
\begin{aligned}
\mathrm{E}\left(S_{X_{1}}\right) & =\sum_{i=1}^{n} \mathrm{E}\left(S_{Y_{i}}\right) i\left(\begin{array}{c}
n \\
i
\end{array}\right) F(u)^{i-1}(1-F(u))^{n-i} \\
& =\frac{n-\sum_{i=1}^{n} \sum_{j=i}^{n}\left(\begin{array}{c}
n \\
j
\end{array}\right) F(u)^{j}(1-F(u))^{n-j}}{\mathrm{E}\left(N\left(u, A, X_{1}\right)\right)} \\
& =\frac{n(1-F(u))}{\mathrm{E}\left(N\left(u, A, X_{1}\right)\right)} .
\end{aligned}
$$

Remark 2. Similar to Remark 1, we can define estimators for $\lambda^{1 / 2}$ based on a sample of durations extracted from the sample paths of the processes $Y_{1}(t), \ldots, Y_{n}(t)$.

The following lemma gives the crossing rate when the number of processes, say $N$, is random.

Lemma 1. Let $X_{j}(t), t \in[0, A], j=1, \ldots, N$, denote some random differentiable processes, where $N$ is a positive integer-valued random variable, independent of the $X_{j}(t)$ 's such that $\operatorname{Pr}(N=n)=p_{n}$ for $n=1,2, \ldots$ with $\sum_{n=1}^{\infty} p_{n}=1$. Define $Y_{1 R}(t)=\min \left\{X_{1}(t), \ldots, X_{N}(t)\right\}$ and $Y_{2 R}(t)=\max \left\{X_{1}(t), \ldots, X_{N}(t)\right\}$, then

(i) $\mathrm{E}\left(N\left(u, A, Y_{1 R}\right)\right)=\sum_{n=1}^{\infty} p_{n} \mathrm{E}\left(N\left(u, A, Y_{1}\right)\right)$

(ii) $\mathrm{E}\left(N\left(u, A, Y_{2 R}\right)\right)=\sum_{n=1}^{\infty} p_{n} \mathrm{E}\left(N\left(u, A, Y_{n}\right)\right)$.

Remark 3. We may use Lemma 1 to calculate the average fade when the wireless communication system receives signals from a large number of propagation systems. In such case, we assume that the $n$ systems are working with probability $p_{n}$.

\section{Excess Height Distribution}

Let $V_{i}$ be the excess height of $Y_{i}(t)$ above a high threshold $u$, i.e., $V_{i}=Y_{i}\left(t_{0}\right)-u$, where $t_{0}$ is a point of the local maximum of $Y(t)$ in $[0, A]$. Since $Y(t)$ is stationary, for ease of discussion and without loss of generality we assume that $t_{0}=0$. Now we are interested to find an approximation to the distribution of $V_{i}$. To accomplish this, we present in the following theorem an approximation to the distribution of $u V_{1}$ following the footsteps of Adler (1981).

Theorem 5. Let $X_{i}(t), i=1, \ldots, n$, denote ergodic and stationary Gaussian processes with $\sigma_{i}^{2}=\operatorname{var}\left(X_{i}(0)\right)$, then as $u \rightarrow \infty$ 
(i) $u V_{1} \stackrel{d}{\rightarrow}$ Exponential $\left(1 / \sum_{i=1}^{n} \sigma_{i}^{-1}\right)$

(ii) $u V_{n} \stackrel{d}{\rightarrow}$ Exponential $\left(1 / \sigma_{(n)}^{2}\right)$,

where $\sigma_{(n)}^{2}=\max \sigma_{i}^{2}$.

Proof. We prove part (ii) and we leave part (i) to the reader. According to Adler (1981, p. 158) we have

$$
\begin{aligned}
\lim _{u \rightarrow \infty}\left(1-F_{u V_{i}}(v)\right) & =\lim _{u \rightarrow \infty} \operatorname{Pr}\left(u V_{i} \geq v \mid Y_{i}(t) \text { has a local maximum }>u \text { at } t=0\right) \\
& =\lim _{u \rightarrow \infty} \frac{\mathrm{E}\left(N\left(u+v / u, A, Y_{i}\right)\right)}{\mathrm{E}\left(N\left(u, A, Y_{i}\right)\right)} .
\end{aligned}
$$

The result follows from Corollary 1 and from the asymptotic formula $1-\Phi(u) \sim \varphi(u) / u$ for $u \rightarrow \infty$.

Corollary 3. If $X_{i}(t), i=1, \ldots, n$, are identically distributed ergodic Gaussian processes, then as $u \rightarrow \infty$

$$
u V_{1} \stackrel{d}{\rightarrow} \text { Exponential }\left(\frac{\sigma^{2}}{n}\right)
$$

Proof. Using equation (6) then for $v>0$ we have

$$
\begin{aligned}
\lim _{u \rightarrow \infty}\left(1-F_{u V_{1}}(v)\right)= & \lim _{u \rightarrow \infty}\left(\frac{\Phi\left(\left(u+\frac{v}{u}\right) / \sigma\right)}{\Phi(u / \sigma)}\right)^{i-1} \times\left(\frac{1-\Phi\left(\left(u+\frac{v}{u}\right) / \sigma\right)}{1-\Phi(u / \sigma)}\right)^{n-i} \\
& \times \frac{\mathrm{E}\left(N\left(u+\frac{v}{u}, A, X_{i}\right)\right)}{\mathrm{E}\left(N\left(u, A, X_{i}\right)\right)} \\
= & \exp \left(-\frac{(i-1) v}{\sigma^{2}}\right) \exp \left(-\frac{(n-i) v}{\sigma^{2}}\right) \exp \left(-\frac{v}{\sigma^{2}}\right) \\
= & \exp \left(-\frac{n v}{\sigma^{2}}\right)
\end{aligned}
$$

Remark 3. Based on $m$ copies of the random variable $V$, we can estimate $\sigma$ using the result in Theorem 5(ii).

\section{Conclusion}

In this paper we defined new random processes, called order statistics processes, as pointwise order statistics of $n$ independent random processes, where $n$ is either a fixed number or an integer-valued random variable. For these new processes, we derived closed form formulas for the upcrossing rates, the cdf of the largest process $Y_{n}(t)$ after an upcrossing of a large level $u$, and the limiting distributions of their excess heights. Furthermore, we derived the average durations of the order statistics process. The motivation to study the order statistics processes is its wide application in wireless-communication systems. Thus, we believe that further work is needed in this topic. For example, Adler (1981) and Adler and Taylor (2007) generalized the notion of upcrossings to random fields. Specifically, they derived an asymptotic formula for the expected number of local maxima of a Gaussian random field above $u$. So an open problem is the asymptotic formulas for the expected number of local maxima by the order statistics of random fields. 


\section{Acknowledgements}

We would like to thank the editor and the referees for their comments which helped us to improve the final structure and the content of this article.

\section{References}

Adler, R. J. (1981). The Geometry of Random Fields. New York: John Wiley and Sons. Adler, R. J., and Taylor, J. E. (2007). Random Fields and Geometry. New York: Springer. Aldous, D. (1989). Probability Approximations via the Poisson Clumping Heuristic. New York: Spring Verlag.

Alodat, M. T., Al-Rawwash, M., and Jebrini, M. (2010). Duration distribution of conjunction of two independent $F$ processes. Journal of Applied Probability, 47, 179-190.

Alodat, M. T., and Anagreh, Y. (2011). Durations distribution of Rayleigh process with application to wind turbines. Journal of Wind Engineering and Industrial Aero dynamics, 99, 651-657.

David, H. A., and Nagaraja, H. (2003). Order Statistics (3rd ed.). Hoboken, NJ: John Wiley and Sons.

Leadbetter, M. R., Lindgren, G., and Rootzén, H. (1983). Extremes and Related Properties of Random Sequences and Processes. New York: Springer Verlag.

Leadbetter, M. R., and Spaniolo, G. V. (2002). On statistics at level crossings by a stationary process. Statistica Neerlandica, 56, 152-164.

Sagias, N. C., Zogas, D. A., and Karagiannidis, G. K. (2005). Selection diversity receivers over non-identical Weibull fading channels. IEEE Transactions on Vehicular Technology, 54, 2146-2151.

Stefanović, M., Panić, S. R., Stefanović, D., Nikolić, B., and Cvetković, A. (2012). Second order statistics of selection combining receiver over $\kappa-\mu$ fading channels subject to co-channel interferences. Radio Science, 47, 1-8.

Worsley, K. J. (1994). Local maxima and expected Euler characteristic of excursion sets of $\chi^{2}, F$ and $t$ fields.

Authors' addresses:

M. T. Alodat

Department of Mathematics, Statistics and Physics

Qatar University

Doha, Qatar

E-mail: alodatmts@yahoo.com

M. Al-Rawwash

Department of Mathematics

Sharjah University

UAE

E-mail: malrawwash@sharjah.ac.ae 